\title{
Reactive Thrombocytosis after Splenectomy in Hereditary Spherocytosis: Case Report and Literature Review
}

\author{
Chidinma Ejikeme ${ }^{1}$, Sherif Elkattawy ${ }^{1}$, Fisayo Kayode-Ajala ${ }^{1}, \underline{\text { Sarah Ayad }}^{1}$, Abraheim Al-nasseri ${ }^{2}$, William Kessler ${ }^{3}$ \\ ${ }^{1}$ Internal Medicine Departent, Rutgers-New Jersey Medical School/Trinitas Regional Medical Center. Elizabeth, NJ, USA \\ ${ }^{2}$ St. George's University, West Indies, Grenada \\ ${ }^{3}$ Department of Hematology and Oncology, Trinitas Regional Medical Center. Elizabeth, NJ, USA
}

\section{Doi: 10.12890/2021_002673 - European Journal of Case Reports in Internal Medicine - @ EFIM 2021}

Received: $27 / 05 / 2021$

Accepted: 30/05/2021

Published: 06/07/2021

\begin{abstract}
How to cite this article: Ejikeme C, Elkattawy S, Kayode-Ajala F, Ayad S, Al-nasseri A, Kessler W. Reactive thrombocytosis after splenectomy in hereditarty
\end{abstract} spherocytosis: case report and literature review. EJCRIM 2021;8: doi:10.12890/2021_002673.

Conflicts of Interests: The authors declare there are no competing interests.

This article is licensed under a Commons Attribution Non-Commercial 4.0 License

\section{ABSTRACT}

Reactive thrombocytosis after splenectomy is a feared cause of thrombosis throughout the arterial and venous system. There are many causes of splenomegaly, ranging from cirrhosis to lymphoma to hereditary spherocytosis. In this report, we will discuss a case of reactive thrombocytosis after splenectomy in a patient with hereditary spherocytosis. Splenomegaly is a relatively common finding in HD patients, causing extravascular haemolysis and thus leading to haemolytic anaemia. Splenectomy is usually considered when patients start to manifest severe symptoms such as abdominal pain, jaundice or worsening liver function tests. Our patient was a good surgical candidate and successfully underwent splenectomy but afterwards developed arterial and venous thrombosis due to reactive thrombocytosis. An extensive hypercoagulable work-up was unremarkable. The patient was started on hydroxyurea and anticoagulation with eventual improvement of platelet levels.

\section{LEARNING POINTS}

- Reactive thrombocytosis can be a significant complication after splenectomy and can range from mild $\left(500,000-700,000 / \mathrm{mm}^{3}\right)$, to moderate $\left(700,000-900,000 / \mathrm{mm}^{3}\right)$, severe $\left(>900,000 / \mathrm{mm}^{3}\right)$ and very severe thrombocytosis $\left(>1,000,000 / \mathrm{mm}^{3}\right)$.

- The use of low-dose hydroxyurea in patients with very severe thrombocytosis can reduce the platelet count to safe levels, and thus, the risk of developing thrombosis.

\section{KEYWORDS}

Thrombocytosis, splenomegaly, hereditary spherocytosis, thrombosis, hypercoagulable

\section{INTRODUCTION}

Hereditary spherocytosis (HS), although very rare, is a major cause of haemolytic anaemia resulting from defects in the red blood cell (RBC) membrane due to variants in genes encoding for membrane or cytoskeleton proteins ${ }^{[1]}$. One of its major complications is splenomegaly, which is treated with splenectomy. We describe the case of a 47-year-old Hispanic woman with a medical history of HS, who developed extensive thrombosis in the splenic vein, superior mesenteric vein (SMV), intrahepatic and extrahepatic portal venous veins as a result of post-splenectomy complications. The decision to perform splenectomy in such patients depends on the severity of the haemolysis, age of the patient and complications of the procedure. Complications of splenectomy include operative infections, venous thromboembolic (VTE) events with thrombocytosis, arterial thrombosis and pulmonary hypertension. VTE complications mostly occur in HS individuals who undergo splenectomy, as in our patient. The relationship between splenectomy in HS patients and reactive thrombocytosis is explored. 


\section{CASE DESCRIPTION}

Our patient was a 47-year-old Hispanic woman with a medical history of HS and chronic anaemia who presented to the emergency department with a 4-day history of abdominal pain. She described the pain as 10/10 in intensity, constant, worse in the left upper quadrant and radiating to the lower back. She denied any alleviating or precipitating factors. She further denied headache, dizziness, chest pain, shortness of breath, nausea, vomiting, diarrhoea or dysuria. Initial vital signs revealed a temperature of $37.8^{\circ} \mathrm{C}$, heart rate of 73 beats per minute, blood pressure of $142 / 74 \mathrm{mmHg}$, respiratory rate of 18 breaths per minute, and oxygen saturation of $100 \%$. On physical examination, the patient had severe diffuse abdominal tenderness. Cardiac and respiratory examinations were unremarkable. A complete blood count was performed, which revealed a white cell count of $21.4(4.7-10.8 \mathrm{~K} / \mu \mathrm{l}), \mathrm{Hb} 8.9(14.0-18.0 \mathrm{~g} / \mathrm{dl})$ and a platelet count of 1,137,000 K/ $\mu$ l (130-400). Computed tomography (CT) of the abdomen was significant for extensive thrombosis in the splenic vein, superior mesenteric vein (SMV), intrahepatic and extrahepatic portal venous veins, as seen in Fig. 1. The patient was started on high-dose heparin infusion and transferred to the ICU for close monitoring.

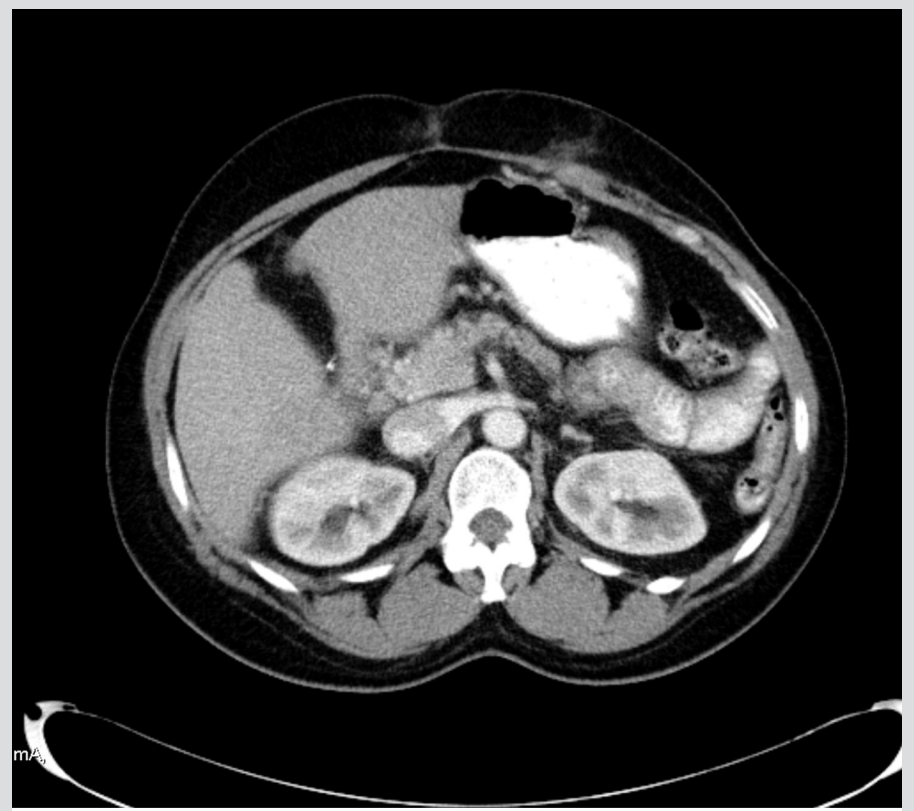

Figure 1. CT scan of the abdomen significant for extensive thrombosis in the splenic vein, superior mesenteric vein (SMV), intrahepatic and extrahepatic portal venous veins

The patient had been diagnosed with HS 'several' years ago. She was followed up periodically in the haematology and oncology clinics where her haemoglobin level, liver function tests and INR were monitored closely. Over time, she started to complain of jaundice, generalized pruritus and scleral icterus. Additionally, her liver enzymes, bilirubin level and INR began to trend upwards. Given worsening liver function, she underwent an open splenectomy with elective cholecystectomy. There were no complications during surgery.

While in the ICU, the patient was started on hydroxyurea for thrombocytosis and morphine for pain. She was also started on total parenteral nutrition to maintain her caloric requirements since her pain worsened with meals. An interventional radiologist was consulted for possible catheter-directed thrombolysis, but recommended delaying the procedure as the risks outweighed the benefits. The platelet count continued to increase, peaking at 1,250,000 K/ $\mu \mathrm{l}$, and eventually started to decline.

A hypercoagulable work-up including protein $\mathrm{C}$ and $\mathrm{S}$, antithrombin III activity, cardiolipin antibody, and factor $\mathrm{V}$ Leiden was performed. Additionally, tests for mutations in the CALR and MPL genes were ordered to rule out other causes of thrombosis. The hypercoagulable work-up was negative, confirming the diagnosis of post-splenectomy thrombosis.

The patient's symptoms started to improve following a week of treatment. Food by mouth was gradually re-introduced. Repeat CT of the abdomen showed stable thrombosis of the splenic vein, SMV, and intrahepatic and extrahepatic portal venous veins. The patient was bridged to coumadin. She was discharged once INR was within the therapeutic range of 2-3. She was recommended to follow up with haematology, surgery, and the medical clinic. Her platelet count had decreased to $458,000 \mathrm{~K} / \mu \mathrm{l}$ at the time of discharge. 


\section{DISCUSSION}

The incidence of haematological and vascular morbidity in post-splenectomy patients is extremely high, with approximately 16,500 patients developing reactive thrombocytosis and 1,100 patients developing thrombosis in the United States each year ${ }^{[1-3]}$. Current research suggests that complications in post-splenectomy patients can be divided into mild thrombocytosis $\left(500,000-700,000 / \mathrm{mm}^{3}\right)$, moderate thrombocytosis $\left(700,000-900,000 / \mathrm{mm}^{3}\right)$, severe thrombocytosis $\left(>900,000 / \mathrm{mm}^{3}\right)$ and very severe thrombocytosis $\left(>1,000,000 / \mathrm{mm}^{3}\right)^{[3-6]}$. Sarbay et al. suggested that treatment is not required in patients who are asymptomatic and have a platelet count below 1,500,000/mm3 after a splenectomy. Further, their research concluded that the use of low-dose hydroxyurea in patients with very severe thrombocytosis can reduce the platelet count to safe levels, and thus, the risk of developing thrombosis ${ }^{[6]}$.

Our patient was a 47-year-old Dominican woman with a medical history of HS who presented to the hospital for an elective laparoscopic splenectomy and cholecystectomy. Successful removal of the spleen and gallbladder was achieved without any peri-operative complications. During post-operative care, the patient was asymptomatic with a platelet count of $891,000 / \mathrm{mm} 3$, and conservative management was provided. Anticoagulation and antiplatelet medications were not given based on the physiological premise that her platelet count should gradually normalize within a few weeks after a splenectomy ${ }^{[7]}$. Additionally, aspirin was not given and hydroxyurea was not given as the patient's platelet count did not exceed $1,500,000 / \mathrm{mm}^{3}$. On post-operation day 7 , the patient remained asymptomatic and was discharged and advised to follow-up with her primary care provider. However, on post-operative day 15 , the patient presented to the ED complaining of abdominal pain with radiation to the back. A CT scan of her abdomen and pelvis was performed and demonstrated thrombosis of her splenic vain, SMV and intra/extra-hepatic venous veins.

This case raises concerns regarding the accuracy of grouping the risk of complications in post-splenectomy patients based on the degree of thrombocytosis. Additionally, the necessity of such grouping is unclear, as specific steps for the management of a patient who meets the criterion of a specific group are lacking. It is possible that the patient in our case may have benefited greatly from anticoagulation and/or antiplatelet therapy even though her platelet count was clearly less than $1,500,000 / \mathrm{mm}^{3}$. The pathophysiological responses to thrombocytosis in patients who have undergone a splenectomy is well-described in the literature ${ }^{[7-88}$. However, Greer et al. concluded that it remains unclear why the platelet count normalizes in the weeks following a splenectomy ${ }^{\left[{ }^{7]}\right.}$. Although the platelet count was normalizing in our patient, physiological control of her platelet count did not suffice. This case suggests that post-operative prophylaxis with anticoagulation and/or antiplatelet medications may have prevented her thrombosis.

\section{CONCLUSIONS}

Thromboprophylaxis at the time of splenectomy should be based on standard practice, but there is no indication for extended thromboprophylaxis beyond the usual duration. While additional research is still needed to ascertain the benefits of prolonged anticoagulation in these patients, our case highlights the possibility of preventing venous thromboembolic events in cases of splenectomy with HS. Additional research is needed to assess more accurately the duration of post-splenectomy prophylaxis and the effectiveness of the anticoagulation and/or antiplatelet medication used to prevent thrombosis.

\section{REFERENCES}

1. Kimmig LM, Palevsky HI. Review of the association between splenectomy and chronic thromboembolic pulmonary hypertension. Ann Am Thorac Soc 2016;13:945-954. doi: 10.1513/AnnalsATS.201512-826FR. PMID: 27058013

2. Boxer MA, Braun J, Ellman L. Thromboembolic risk of postsplenectomy thrombocytosis. Arch Surg1978;113:808-809. doi: 10.1001/archsurg.1978.01370190030004

3. Stamou KM, Toutouzas KG, Kekis PB, Nakos S, Gafou A, Manouras A, et al. Prospective study of the incidence and risk factors of postsplenectomy thrombosis of the portal, mesenteric, and splenic veins. Arch Surg 2006;141:663-669. doi: 10.1001/archsurg.141.7.663. PMID: 16847237.

4. Chiarello P, Magnolia M, Rubino M, Liguori SA, Miniero R. Thrombocytosis in children. Minerva Pediatr 2011;63:507-513.

5. Yadav D, Chandra J, Sharma S, Singh V. Clinicohematological study of thrombocytosis. Indian J Pediatr 2010;77:643-647. doi: 10.1007/s12098-010-0091

6. Sarbay H, Akbayram S. Secondary severe thrombocytosis in a patient who underwent splenectomy due to hereditary spherocytosis and its treatment using hydroxyurea. Pan Afr Med J 2019;32:175. doi: 10.11604/pamj.2019.32.175.17841

7. Greer JP, Foerster J, Lukens JN, Rodgers GM, Paraskevas F, Glader B, editors. Wintrobe's Clinical Hematology. 11th ed. Philadelphia: Lippincott Williams \& Wilkins; 1981. pp. 1128-1134.

8. Mentzer WC. Hereditary spherocytosis. UpToDate 2021. 